\title{
Diurnal rhythmic expression of the rhythm-related genes, rPeriod1, rPeriod2, and rClock, in the rat brain
}

\author{
Kun-Ruey Shieh ${ }^{1,2,4, *}$, Shu-Chuan Yang ${ }^{2,3}$, Xin-Yun $\mathrm{Lu}^{4}$, Huda Akil ${ }^{4}$ \& Stanley J. \\ Watson ${ }^{4}$ \\ ${ }^{1}$ Institute of Neuroscience and ${ }^{2}$ Department of Physiology, Tzu Chi University, Hualien 970, ROC; \\ ${ }^{3}$ Department of Nursing, Tzu Chi College of Technology, Hualien 970, ROC; ${ }^{4}$ Mental Health Research \\ Institute, The University of Michigan School of Medicine, Ann Arbor, MI 48109, USA
}

Received 14 June 2004; accepted in revised form 1 September 2004

(C) 2005 National Science Council, Taipei

Key words: arcuate nucleus, cerebellum, circadian rhythm, hippocampus, pars tuberalis/median eminence, suprachiasmatic nucleus

\section{Summary}

High densities of the mRNA of three rhythm-related genes, rPeriod1 (rPer1), rPer2, and rClock, which share high homology in Drosophila and mammals, are found in the rat hypothalamic suprachiasmatic nucleus $(\mathrm{SCN})$. The SCN, however, is not the only brain region that expresses these genes. To understand the possible physiological roles of these rhythm-related genes, we examined expression of these genes in different brain regions at various time points in male Sprague-Dawley rats. Using semi quantitative in situ hybridization with ${ }^{35} \mathrm{~S}$-riboprobes to evaluate mRNA levels, the diurnal rhythmicity of $r$ Perl 1 , and $r$ Per 2 mRNA levels was found in the SCN, arcuate nucleus, and median eminence/pars tuberalis. Expression patterns of mRNA for $r$ Per 1 and $r$ Per 2 , however, were not similar in these brain regions. The rhythmicity in these brain regions was specific, because it was not observed in the cerebellum or hippocampus. Moreover, diurnal changes in $r$ Clock mRNA expression were not detected in any of the brain regions examined. These findings suggest that the different expression patterns observed for $r$ Per $1, r$ Per 2 , and $r$ Clock mRNAs may be attributed to their different physiological roles in these brain regions, and support previous work indicating that circadian rhythms in the brain are widespread.

\section{Introduction}

Most, if not all, eukaryotes have internal timing mechanisms which generate physiological rhythms that can be synchronized to the light-dark cycle of the earth. The hypothalamic suprachiasmatic nucleus $(\mathrm{SCN})$ is the principal circadian pacemaker that drives vigorous oscillations in a wide range of vital physiological processes [1]. Recently, rhythmrelated genes in the SCN such as Period (Per) 1,2, 3, Clock, Bmal1, Timeless, and Cryptochrome1, 2

*To whom correspondence should be addressed. Fax: 886-38461733; e-mail: krishieh@mail.tcu.edu.tw have been discovered, and shown or proposed to exert circadian phenomena in the SCN through the relations of these genes to feedback loops centered on a transcriptional/translational cycle [2-5]. A number of reports have suggested that Perl is a clock-oscillating gene. First, transcripts of Per 1 show a very robust rhythm in the SCN of mice $[6,7]$ and rats [1], even in constant dark (DD) conditions [8]. Additionally, negative and positive elements necessary for closing the auto-feedback loop of the Perl gene and the Drosophila Period gene system have been delineated [9]. In one study, the transcription of Perl in mice was shown to be 
activated by the binding of the CLOCK (the product of Clock gene)-BMAL1 complex to the Eboxes in the promoter region of the Perl gene [10]; this activation is specifically inhibited by PER1 protein (the product of Perl gene) and other negative elements including PER2 (the product of Per2 gene), PER3, TIM, CRY1, and CRY2 [1114]. Moreover, Per 1 mRNA is rapidly induced by light in the mouse SCN, and it correlates well with the resetting of locomotor activity [8]. Our previous study showed that rPerl, rPer2, and rClock mRNAs exhibit strong expression throughout the SCN [15]. Yan and colleagues [16, 17] demonstrated that rPerl and rPer2 mRNAs in the ventrolateral SCN can be induced by acute light. These findings suggest that circadian rhythms can be regulated by different signals from different subdivisions of the SCN [18-20].

The SCN, however, is not the only brain region that expresses rhythm-related genes, and neither is it the sole area that exhibits circadian expression of these genes [7, 15, 17, 21-33]. The physiological roles of extra-SCN brain regions with circadian regulation are still poorly understood. Recently, Per 1 and Per 2 mRNAs in the median eminence/ pars tuberalis (ME/PT) of seasonally breeding animals have been demonstrated to be related to prolactin secretion [21, 30, 34-37]. Delineating the circadian profiles of rhythm-related genes in different brain regions will help elucidate the physiological roles of these genes. In this study, we examined the expression of rPerl, rPer2, and rClock mRNAs in the $\mathrm{SCN}$, arcuate nucleus (ARC), ME/PT, cerebellum, and gyrus dentatus (DG) and CA1 of Ammon's horn of the hippocampus in the rat brain.

\section{Experimental procedures}

\section{Animals}

Adult male Sprague-Dawley rats $(n=48)$, weighing between 300 and $350 \mathrm{~g}$, were purchased from the National Experimental Animal Breeding and Research Center of the National Science Council (Taipei, Taiwan), and were housed two per cage in a temperature $\left(22 \pm 1{ }^{\circ} \mathrm{C}\right)$ - and light (lights on from 0600 to $1800 \mathrm{~h}$ )-controlled room with free access to rat chow and tap water. Animals were habituated to the housing conditions for at least
1 week before the experiments, and were treated according to the guidelines of the Institutional Animal Care and Use Committee of Tzu Chi University. We also minimized the number of animals used as much as we could. Rats were sacrificed by rapid decapitation every $4 \mathrm{~h}$ from lights on, which was identified as the Zeitgeber time 0 (ZT $0)$. To eliminate the transient effect of light-dark switching on rats, we also sacrificed animals at ZT 11.5 and ZT 23.5. The brains were removed, frozen in isopentane $\left(-40{ }^{\circ} \mathrm{C}\right)$ to minimize degradation of the mRNAs, and stored at $-80{ }^{\circ} \mathrm{C}$. The frozen brains were sectioned using a cryostat. Thin $(20 \mu \mathrm{m})$, serial coronal brain sections were cut, then thaw-mounted onto polylysine-subbed glass slides, and stored at $-80{ }^{\circ} \mathrm{C}$ until used.

\section{In situ hybridization histochemistry}

Three to five frozen slides (four sections/slide) per rat brain were selected for in situ hybridization. The coordinates of the SCN, ARC, ME/ PT, hippocampus, and cerebellum were bregma -0.9 to $-1.5,-2.3$ to $-3.3,-2.3$ to $-3.6,-2.8$ to $-4.1,-10.0$ to $-10.5 \mathrm{~mm}$, respectively. Briefly, slides were removed from a $-80{ }^{\circ} \mathrm{C}$ freezer, fixed in $4 \%$ paraformaldehyde for $1 \mathrm{~h}$, and rinsed three times in $2 \times$ SSC $(1 \times$ SSC: $0.15 \mathrm{M} \mathrm{NaCl}$, $0.015 \mathrm{M}$ Na citrate, $\mathrm{pH}$ 7.2). Then the sections were placed in a solution containing acetic anhydrate $(0.25 \%)$ in triethanolamine $(0.1 \mathrm{M}, \mathrm{pH}$ 8.0) at room temperature for $10 \mathrm{~min}$, rinsed in distilled water, dehydrated through a graded ethanol series $(50,70,80,95$, and $100 \%)$, and subsequently air-dried.

The ${ }^{35} \mathrm{~S}$-labeled antisense cRNA probes for rPer1 (581-1546), rPer2 (2482-3576) and rClock (1361-2027) were in vitro transcripted by the T3 and $\mathrm{T} 7$ polymerase, prepared at $37^{\circ} \mathrm{C}$, then processed for in situ hybridization as described previously [15]. To ensure comparability, slides from different time points were processed with the same hybridization. Briefly, brain sections were hybridized overnight with both probes at $55^{\circ} \mathrm{C}$ as previously described [15]. After hybridization, coverslips were removed, and slides were rinsed 3 times in $2 \times \mathrm{SSC}$, and incubated in RNase A $(200 \mu \mathrm{g} / \mathrm{ml})$ at $37^{\circ} \mathrm{C}$ for $1 \mathrm{~h}$. Slides were washed sequentially with $2 \times \mathrm{SSC}, 1 \times \mathrm{SSC}, 0.5 \times \mathrm{SSC}$, and $0.1 \times \mathrm{SSC}$ for $5 \mathrm{~min}$ and $0.1 \times \mathrm{SSC}$ at $65^{\circ} \mathrm{C}$ for $1 \mathrm{~h}$, then rinsed in distilled water, dehydrated 
through graded ethanol $(50,70,80,95$, and $100 \%)$, and air-dried.

\section{Semiquantitative analysis of radioactive in situ hybridization autoradiography and statistical analysis}

To ensure comparability of the slides from the same brain regions among different time points, slides from the same brain regions were exposed on the same film, or each cassette contained slides of all eight time points (ZT 0, 4, 8, 11.5, 12, 16, 20, and 23.5). After exposure to Kodak BioMax film (Kodak, Rochester, NY, USA) for 2-7 days, the images of sections were captured and digitized with a Pixera CCD video camera (Penguin 150CL, Pixera Corp., Los Gatos, CA, USA), and then the intensity of the hybridization signal in the whole brain was assessed against a ${ }^{14} \mathrm{C}$ curve using NIH Image software.

Images were subjected to densitometric analysis, yielding measurements of the relative optical density (ROD) and expression area. The intensity values in the corpus callosum were subtracted as the background. The ROD, which assesses the mean gray value above the threshold (mean of background plus $3.5 \times$ the standard deviation) per pixel of the measured area, was used to quantify the intensity of the signal. The expression area was the number of pixels above the threshold within the defined brain regions. Images were measured from 5 to 8 sections for each animal at every time point. The specificity of hybridization was ascertained by hybridization with sense strand probes or pretreatment with RNase $(200 \mu \mathrm{g} / \mathrm{ml}$ at $37{ }^{\circ} \mathrm{C}$ for $60 \mathrm{~min}$ ) before hybridization as previously described [15]. The expression of rPerl, $r$ Per 2 , and $r$ Clock mRNA was not distinguishable before or after the light-dark switch (ZT 11.5 vs. ZT 12 and ZT 23.5 vs. ZT 0), thus the results at ZT 11.5 and 12 and at ZT 23.5 and 0 were pooled, respectively. One-way ANOVA was used to test the significance of differences among groups. A value of $p<0.05$ was considered statistically significant.

\section{Results}

Diurnal patterns of rPerl and rPer2, but not $r$ Clock, mRNA in rat brain regions, including the hypothalamic SCN, ME/PT, and ARC were analyzed by in situ hybridization using ${ }^{35}$ S-labeled antisense RNA probes. To confirm the specificity of the in situ hybridization signals, we used the sense rPerl, rPer2, and rClock RNA probes or RNase-preincubated antisense RNA probes for the control study as previously described [15]. These data support the specificity of in situ hybridization in this study.

\section{Expression patterns of rPer $1 \mathrm{mRNA}$}

The highest expression of rPerl mRNA in the SCN was found at ZT 4 and showed a diurnal pattern using the ROD and expression area as the indices of mRNA signals $(p<0.01$; Figure 1c). rPerl mRNA expression initiated from the dorsomedial part of the SCN (dmSCN) at ZT 4, and had extended to the ventrolateral part (vlSCN) by ZT 8 and 12 (Figure 1b). A diurnal pattern of rPer 1 mRNA was also observed in the ME/PT ( $p<0.01$; Figure 1f). However, the pattern in the ME/PT differed from that in the SCN. rPer $1 \mathrm{mRNA}$ in the ME/PT at ZT 0 exhibited the highest levels in the parameters of ROD and expression area, and a moderate level of rPer 1 mRNA in the ME/PT was revealed at ZT 12 using the expression area as the index, but not using the ROD $(p<0.01$; Figure 1f). Images of rPerl mRNA in the ME/PT are shown in Figure 2e. Additionally, a diurnal pattern of rPerl mRNA was also found in the ARC. The highest expression areas, excluding the ROD, of $r$ Perl mRNA in the ARC were detected at ZT $12(p<0.01$; Figure $1 \mathrm{~h}$ and i). Nevertheless, a diurnal pattern of rPerl mRNA was not shown in all brain regions. Neither the hippocampal CA1 and DG nor the cerebellum exhibited rhythmic expression of rPerl mRNA using both the ROD and the expression area as indices (Figure $3 \mathrm{a}-\mathrm{c}$ ).

\section{Expression patterns of $r$ Per $2 \mathrm{mRNA}$}

The highest levels of rPer2 mRNA in the SCN were detected at ZT 8 and 12, and a diurnal pattern was exhibited according to the ROD and expression area $(p<0.01$; Figure 2c). The spatial expression of $r$ Per 2 mRNA was similar to that of rPerl, which initiated from the $\mathrm{dmSCN}$ at ZT 4 , and then had extended to the vlSCN by ZT 8 and 12 (Figure $2 b$ ). A diurnal pattern of $r$ Per 2 mRNA 
(a)

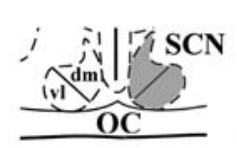

(d)

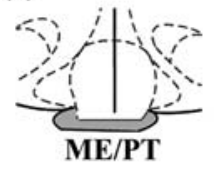

(g)

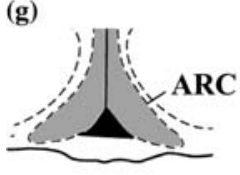

(b)

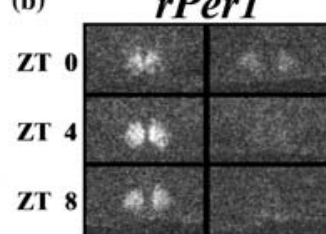

(e)

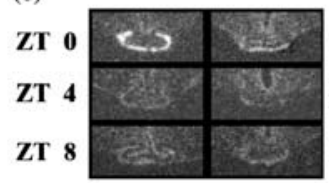

(h)

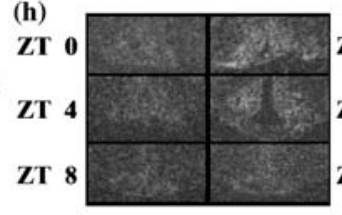

(c)
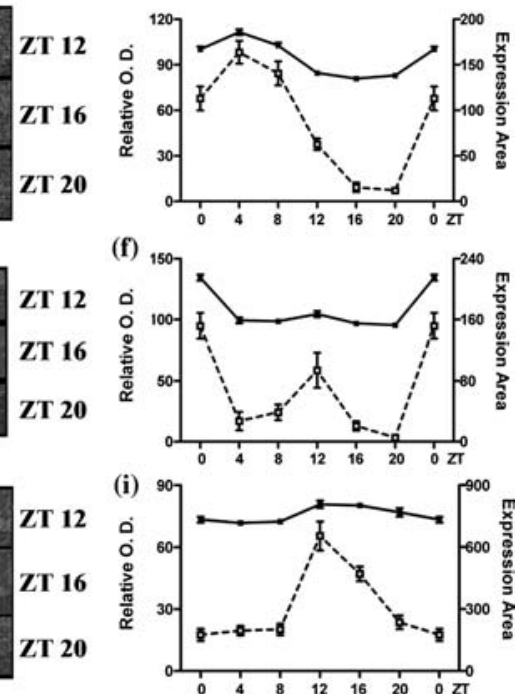

Figure 1. Diurnal patterns of rPer 1 mRNA using the ROD (relative optical density, filled squares with a solid line) and expression area (open squares with a dotted line) as indices in the rat SCN (panels a-c), ME/PT (panels d-f), and ARC (panels g-i). Rats were sacrificed every $4 \mathrm{~h}$ after lights on (ZT 0). The vertical lines at each time point represent the SE $(n=5-6)$. Panels a, d, and g, respectively, represent the schematic diagrams for the $\mathrm{SCN}, \mathrm{ME} / \mathrm{PT}$, and $\mathrm{ARC}$. Panels b, e, and h, respectively, represent the photomicrographs of rPerl mRNA signals in the SCN, ME/PT, and ARC, from ZT 0 to ZT 20.

(a)

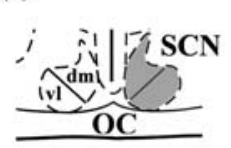

(d)

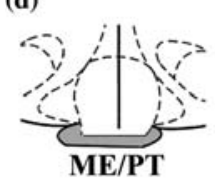

(g)

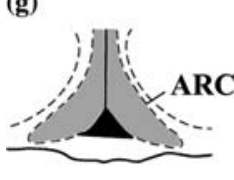

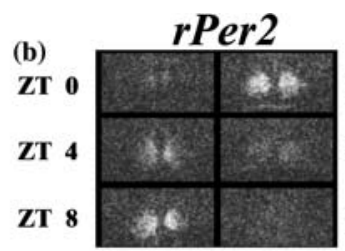

(e)

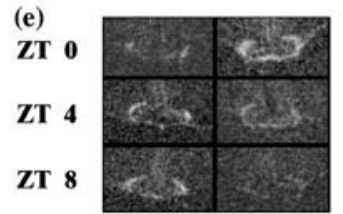

(h)

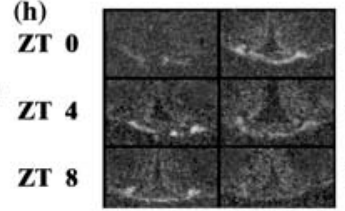

(c)
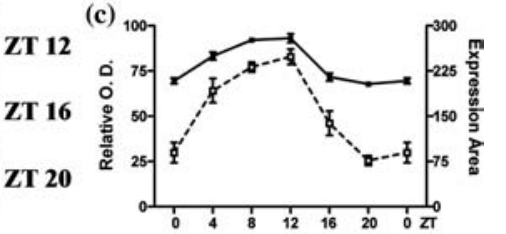

ZT 12
ZT 16
ZT 20

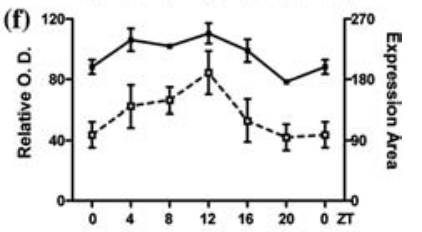

ZT 12
ZT 16
ZT 20

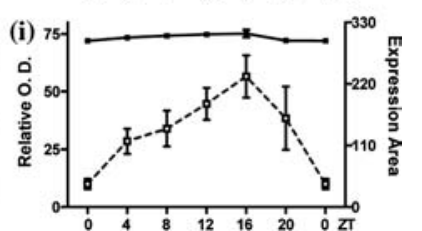

Figure 2. Diurnal patterns of rPer 2 mRNA using the ROD (filled squares with a solid line) and expression area (open squares with a dotted line) as indices in the rat SCN (panels a-c), ME/PT (panels d-f), and ARC (panels g-i). Rats were sacrificed every 4 h after lights on (ZT 0). The vertical lines at each time point represent the SE $(n=5-6)$. Panels a, d, and g, respectively, represent the schematic diagrams for the SCN, ME/PT, and ARC. Panels b, e, and h, respectively, represent the photomicrographs of $r$ Per 2 mRNA signals in the SCN, ME/PT, and ARC, from ZT 0 to ZT 20.

was also found in the ME/PT $(p<0.05$; Figure 2f) and was similar to that seen in the SCN (Figure 2c); the gradient increased from ZT 0 and reached a peak at ZT 8-12 (Figure 2c and f).
Images of $r$ Per 2 mRNA in the ME/PT are shown in Figure 3e. A diurnal pattern of rPer2 mRNA was also found in the ARC by using the expression area, but not ROD, as the index $(p<0.01$; 

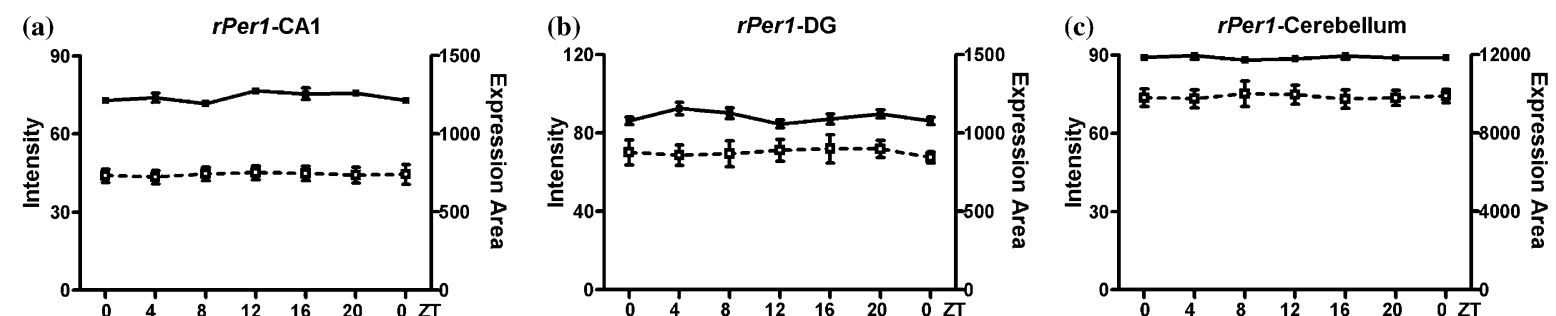

(d)

(e)
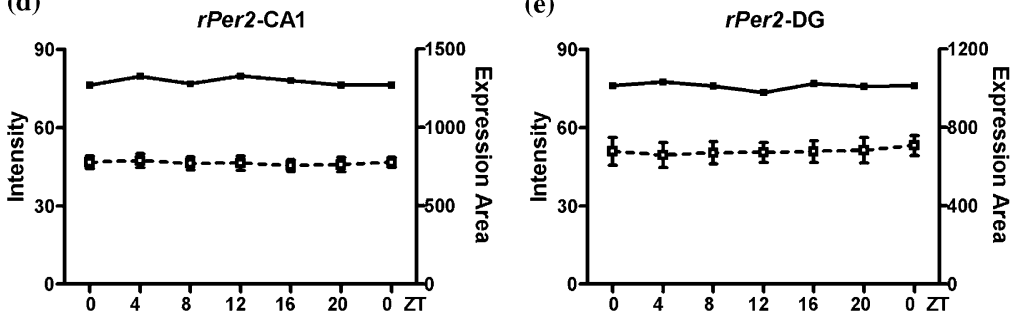

(f)

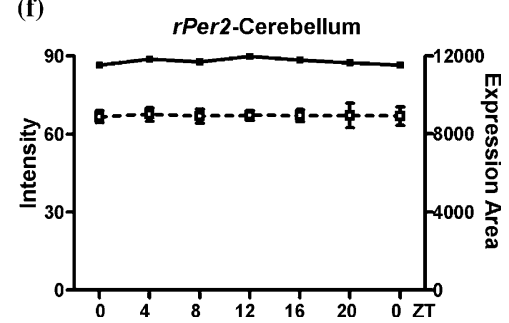

Figure 3. Expressions of $r$ Perl (panels a-c) and rPer2 (panels d-f) mRNA using the ROD (filled squares with a solid line) and expression area (open squares with a dotted line) as indices in the rat CA1 (panels a and d) and DG (panels b and e) of the hippocampus, and cerebellum (panels $\mathrm{c}-\mathrm{f}$ ). Rats were sacrificed every $4 \mathrm{~h}$ after lights on (ZT 0). The vertical lines at each time point represent the SE $(n=5-6)$.
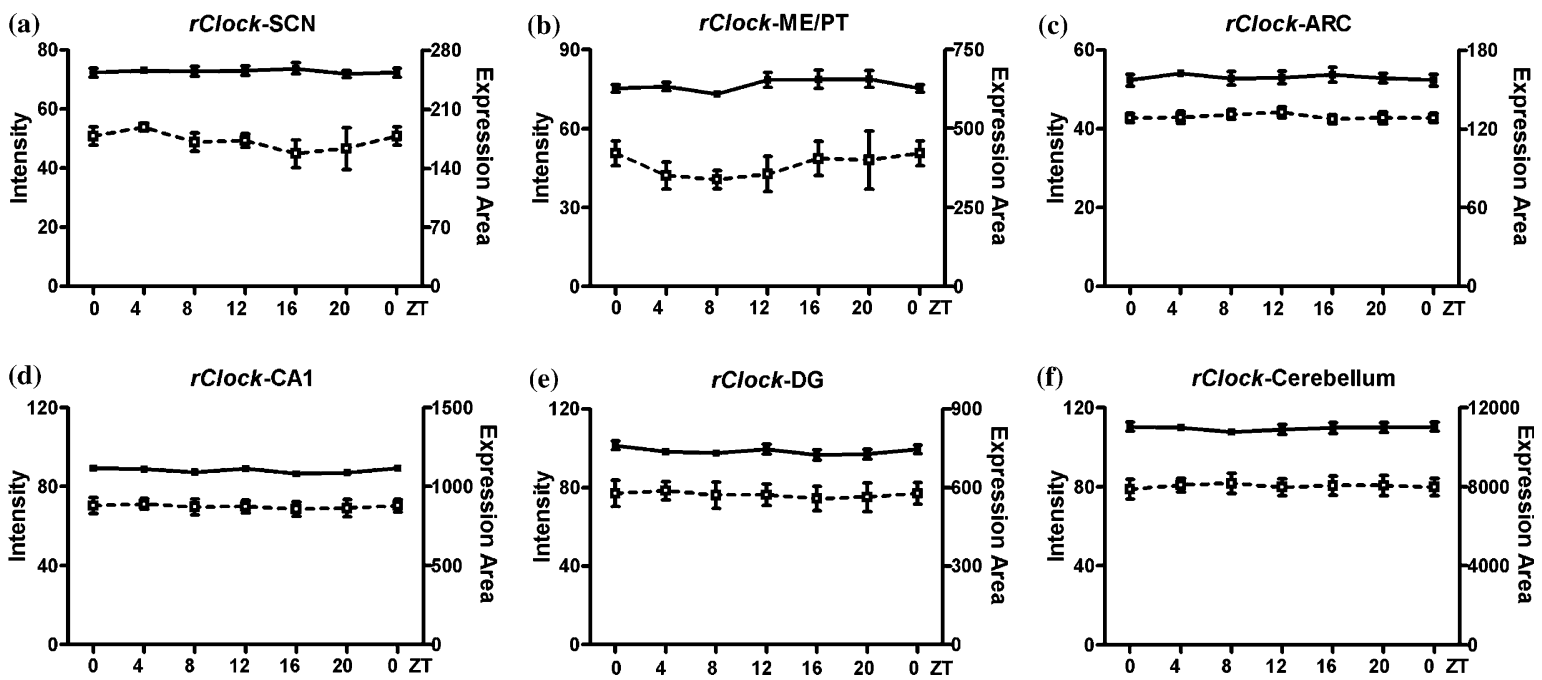

Figure 4. Expression of $r$ Clock mRNA using the ROD (filled squares with a solid line) with or without the expression area (open squares with a dotted line) as indices in the rat SCN, ME/PT, ARC, the CA1 and DG of the hippocampus, and cerebellum, respectively (panels a-f). Rats were sacrificed every $4 \mathrm{~h}$ after lights on (ZT 0). The vertical lines at each time point represent the SE $(n=5-6)$.

Figure 2i). The highest levels of the rPer2 mRNA expression area in the ARC were detected between ZT 12 and ZT16 ( $p<0.01$; Figure 2i). Similar to $r$ Perl, the rhythmic pattern of rPer 2 mRNA was not found in the DG and CA1, or the cerebellum by using both the ROD and expression area as the indices (Figure 3d-f).

\section{Expression patterns of $r$ Clock $m R N A$}

The expression of $r$ Clock mRNA in the SCN, ME/ PT, ARC, hippocampal CA1 and DG, and cerebellum did not exhibit a rhythmic pattern by using both the ROD and expression area as the indices (Figure 4a-f). 


\section{Discussion}

Diurnal patterns of rPer 1 and rPer $2 \mathrm{mRNA}$ expression in the rat $S C N, M E / P T$, and $A R C$

In the present study, we not only examined the topographic expressions of rPerl, rPer2, and $r$ Clock mRNA but also showed the diurnal profiles of these genes in several rat brain regions, including the SCN, ARC, and ME/PT by semi-quantitative in situ hybridization. In addition, we demonstrated that these genes did not exhibit rhythmicity in the hippocampus or cerebellum. Using the ROD and expression area as indices for gene expression, we found that rPerl mRNA expression originated from the $\mathrm{dmSCN}$ at ZT 0 , and had extended to the vlSCN by ZT 4 (Figure 1b). The intensity of rPerl mRNA in the dmSCN was reduced at ZT 8, but was still exhibited at similar levels in the $\mathrm{dmSCN}$ and vlSCN (Figure 1b). The rPerl signal in the entire SCN had become faint by ZT 12, and was almost undetectable at ZT 16 and ZT 20. A similar expression pattern was observed for $r$ Per 2 mRNA, but with a lag of around $4 \mathrm{~h}$ when compared to rPer 1 mRNA expression, i.e., initiation in the $\mathrm{dmSCN}$ was found at ZT 4 (Figure 1b). Using digoxigenin-labelled probes, Yan and Okamura [16] showed that the circadian profiles of rPerl and rPer 2 mRNA expression initiated from the periventricular part of the $\mathrm{dmSCN}$, then spread to the central part of the dmSCN, and finally extending to the vlSCN. Similar results were found in the present study using isotope-labelled probes. We not only confirmed the findings of Yan and Okamura [16], but also attempted to examine the subtle expression profiles of rhythm-related genes in different subdivisions of the SCN using different indices including the ROD and expression area. Our data support the previous finding of Yan and Okamura [16] that rhythm-related gene expressions in brain regions possess the topographic characteristics of neurotransmitters and neuropeptides. Additionally, a diurnal pattern of $r$ Perl mRNA in rat suprachiasmatic nucleus was also found in a recent study [38].

Diurnal patterns of Per 1 and Per 2 mRNA in the $\mathrm{ME} / \mathrm{PT}$ of seasonally breeding animals (sheep and hamsters) were demonstrated in previous studies [34-37, 39]. In this study, we found that rPerl and rPer 2 mRNA in the rat ME/PT showed diurnal profiles. Higher-expression areas of $r$ Perl mRNA in the ME/PT were found at ZT 0 and ZT 12 , although the highest signal intensity was only found at ZT 0 (Figure 1e, f). Our findings slightly differ from those of previous reports in sheep and hamsters which revealed that the highest intensity of Per 1 mRNA occurred at ZT 23 and ZT 3 with the short photoperiod, and only at ZT 3 with the long photoperiod, respectively [34-36, 39]. In agreement with the previous studies in sheep and hamsters, the highest $r$ Per 1 mRNA signal in the rat $\mathrm{ME} / \mathrm{PT}$ was found at ZT 0 (between ZT 23 and ZT $3)$. In contrast, an expression area with a moderate signal of $r$ Per 1 mRNA in the rat $\mathrm{ME} / \mathrm{PT}$ was also found at ZT 12. To exclude the transient possibility of the light-dark switch, we examined the expression of mRNAs at ZT11.5 and ZT 12 and found no difference between these two time points in any brain region (data not shown). Furthermore, a recent study [40] showed a similar pattern of $r$ Per 1 mRNA, i.e., highest at ZT 1 and moderate at ZT 12 in the rat $\mathrm{ME} / \mathrm{PT}$, and proposed that this diurnal pattern may be driven by melatonin. Diurnal expression of rPer $2 \mathrm{mRNA}$ in the rat ME/PT was also found in this study. The highest levels of ROD and expression area of the rPer 2 mRNA signal were detected at ZT 12, and the lowest at ZT 20 (Figure 2f). The diurnal pattern of rPerl mRNA differed from that of $r$ Per 2 mRNA (Figures $1 \mathrm{f}$ and $2 \mathrm{f})$. This indicated that the expression of $r$ Perl and rPer 2 mRNA might be due to regulation by tissuespecific rhythmic properties of physiological function. Relationships among Perl mRNA, melatonin, and prolactin in seasonally breeding animals were delineated in previous studies [34, 37, 39]. A diurnal pattern of rPer 2 mRNA expression in sheep $\mathrm{ME} / \mathrm{PT}$ has been reported, with a peak found at ZT 7 in the long photoperiod and at ZT 3 in the short photoperiod [35]. Due to differences of the light-dark duration and species, the peak of $r$ Per 2 mRNA expression in this study differed from that of the previous study [35]. Whether rPer 2 mRNA in the $\mathrm{ME} / \mathrm{PT}$ is regulated by melatonin, as is $r$ Per 1 mRNA, remains to be investigated.

In addition to the $\mathrm{SCN}$ and $\mathrm{ME} / \mathrm{PT}$, a third region found to exhibit a diurnal rhythm of $r$ Perl and rPer 2 mRNA was the hypothalamic ARC (Figures $1 \mathrm{i}$ and $2 \mathrm{i}$ ). The highest and then moderate expressions of rPer 1 mRNA in the ARC were found at ZT 12 and at ZT 16, respectively (Figure $1 \mathrm{~h}, \mathrm{i})$. These results are similar to recent 
studies using Perl-luciferase transgenic rats [41, 42]. Results from brain slice cultures of Perlluciferase transgenic rats indicated that the ARC has its own in vitro rhythmicity [41]. Kriegsfeld et al. [42] showed that the number of cells with Per 1 mRNA and PER1 signals in the ARC of female Per $1:$ jellyfish green fluorescent protein (GFP) transgenic mice had significantly increased by ZT 10 and decreased by ZT 22. Moreover, diurnal expression of $r$ Per $2 \mathrm{mRNA}$ was also found in this study. The highest expression area of the $r$ Per 2 mRNA signal in the rat ARC was observed at ZT 16 (Figure $2 \mathrm{~h}$ and i). To the best of our knowledge, the diurnal pattern of rPer 2 mRNA expression in the rat ARC has not been previously reported. However, determining the physiological role of $r$ Per 2 mRNA expression in the rat ARC requires further studies.

Comparison of rPer 1 and $r$ Per $2 m R N A$ expression patterns among the $S C N, M E / P T$, and $A R C$

Since multiple oscillators in the brain have been found in vitro, the theory of a major pacemaker in the SCN has been challenged. However, the existence of multiple oscillators in different brain regions does not mean that the SCN has lost its ascendancy in the regulation of circadian rhythmicity. Whether the SCN still plays a role as the dominant driver in regulating the circadian rhythm is interesting to chronobiologists. In this study, we compared the rhythmic profiles of $r$ Per 1 and $r$ Per 2 mRNA expression among the SCN, ARC, and $\mathrm{ME} / \mathrm{PT}$, and tried to find the possible relationships among these brain regions. We found that the highest expressions of rPer 1 mRNA in the $\mathrm{SCN}$, $\mathrm{ARC}$, and ME/PT occurred at ZT 4, ZT 12, and ZT 12, respectively. The rhythmicity of rPerl mRNA in the ARC at ZT 12 was highly correlated to that in the $\mathrm{ME} / \mathrm{PT}$ at the same time point. Kriegsfeld et al. [42] showed that the number of cells with Per 1 mRNA and PER1 signal and the percentage of tyrosine hydroxylase cells containing GFP or PER 1 in the ARC of female Per $1::$ GFP transgenic mice were significantly higher at ZT 10 and lower at ZT 22. This finding implied that tuberoinfundibular dopaminergic (TIDA) neuronal activity is associated with Perl mRNA expression and regulates the pituitary's secretion of prolactin. However, our previous studies showed that the diurnal rhythm of TIDA neuronal activity is only exhibited in female but not in male animals, either those intact or with estrogen replacement $[43,44]$. This diurnal rhythm of TIDA neuronal activity is higher before ZT 14 and lower after ZT 14 only in female rats, and not in male rats [43-45]. In this study, we found that the diurnal expression of rPer $1 \mathrm{mRNA}$ still existed in the ARC of male rats and showed higher expression at ZT 12. Therefore, whether the diurnal rhythm of rPerl mRNA in the ARC of male rats is involved in the regulation of TIDA systems is uncertain. In addition, $r$ Per 1 mRNA was not correlated well at ZT 0 , i.e., when the highest expression in the ME/PT and the lowest in the ARC were found. Taken together, a previous study in mice [42] and this study in rats show that the peak of the Per 1 mRNA signal in the ARC is found at ZT 12. The peak of the Perl mRNA signal in the ME/PT in hamsters and sheep [34-37, 39], and in rats (the present study) occurred at around ZT 23-3 and ZT 12. Therefore, the correlation of Per 1 mRNA expression between the $\mathrm{ARC}$ and $\mathrm{ME} / \mathrm{PT}$ is poor, and further studies are needed to resolve this puzzle.

In $r$ Per 2 mRNA expression profiles, peaks in the SCN, ARC and ME/PT were found at ZT 812, ZT 12-16, and ZT 12, respectively. The expression pattern of $r$ Per 2 mRNA in the ME/PT was similar to that in the SCN. The expression pattern of $r$ Per 2 mRNA in the ARC was $4 \mathrm{~h}$ behind those in the SCN and ME/PT. An in vitro study using Per2-luciferase transgenic animals may help elucidate the relationships among the $\mathrm{SCN}$, ARC, and ME/PT. Interestingly, the expression profile of rPerl mRNA in this study was around $4 \mathrm{~h}$ ahead of that of $r$ Per 2 mRNA in both the SCN and ARC. This similarity, however, was not found between the SCN and ME/PT.

In addition to measuring rPerl and rPer2 mRNA expressions in the SCN, ARC, and ME/PT, we also evaluated their expressions in the DG and CA1 of the hippocampus, and in the cerebellum. Similar to a previous in vitro study [41], we did not find a diurnal pattern of $r$ Per 1 and $r$ Per 2 mRNA signals in the rat cerebellum or hippocampus. Moreover, we also found no rhythmicity of $r$ Clock gene expression in the $\mathrm{SCN}, \mathrm{ARC}, \mathrm{ME} / \mathrm{PT}$, the DG and CA1 of the hippocampus or cerebellum. This confirms previous findings which demonstrated that Clock is constitutively expressed in different brain areas, although it has been shown to be necessary for circadian rhythm generation [46]. 
In the present study we used the ROD and expression area to measure rhythm-related gene expression levels, and tried to obtain more objective information about the circadian profiles in these genes. Our data indicated that the expression area is a more sensitive index than ROD. In fact, the meaning of ROD and expression area measurements is subtly different because the changes of gene expression can be induced by two possible modalities. One is from the expression level in each cell or brain region, and the other is from the cell numbers. The ROD belongs to the former and the expression area is the latter modality. Nevertheless, sometimes it is not easy to distinguish these two situations. Therefore, some researchers prefer to use the integrative optical density (IOD), which is the expression area times density [47], or only use the ROD to present the data [23]. However, quantifying the expression area also has its limitations. One of the major limitations is the coordinates of brain regions. The other is that the selection of a quantification region is subjective and therefore causes bias. Thus, although we cannot rule out artifacts and definitively establish that these rhythm-related gene expressions outside of the SCN have rhythmic changes in cell numbers or gene contents, this study still supports that the expression of these rhythm-related genes in the brain regions of the rat have topographic characteristics.

In conclusion, the present study has shown that the diurnal patterns of $r P e r 1$ and $r P e r 2$ mRNA are extensive and region-specific. However, some differences in the expression patterns were found among these rhythm-related genes, which can be possible targets for investigations of the physiological roles of these genes in different brain regions.

\section{Acknowledgements}

We are grateful to Prof. Ted H. Chiu for a helpful and careful review of this manuscript. We also thank Mr. D.P. Chamberlin for editorial assistance. This study was supported in part by the National Science Council in Taiwan, ROC (NSC 91-2320-B-320-004, NSC 92-2320-B-320-021, and NSC 93-2320-B-320-010) and a Tzu Chi University Research Grant (TCMRC9314A) to KunRuey Shieh.

\section{References}

1. Hastings M. and Maywood E.S., Circadian clocks in the mammalian brain. Bioassays 22: 23-31, 2000.

2. Dunlap J.C., Molecular bases for circadian clocks. Cell 96: 271-290, 1999.

3. Reppert S.M. and Weaver D.R., Coordination of circadian timing in mammals. Nature 418: 935-941, 2002.

4. Wager-Smith K. and Kay S.A., Circadian rhythm genetics: from flies to mice to humans. Nat. Genet. 26: 23-27, 2000.

5. Young M.W. and Kay S.A., Time zones: a comparative genetics of circadian clocks. Nat. Rev. Genet. 2: 702-715, 2001.

6. Sun Z.S., Albrecht U., Zhuchenko O., Bailey J., Eichele G. and Lee C.C., RIGUI, a putative mammalian ortholog of the Drosophila period gene. Cell 90: 1003-1011, 1997.

7. Tei H., Okamura H., Shigeyoshi Y., Fukuhara C., Ozawa R., Hirose M. and Sakaki Y., Circadian oscillation of a mammalian homologue of the Drosophila period gene. Nature 389: 512-516, 1997.

8. Shigeyoshi Y., Taguchi K., Yamamoto S., Takekida S., Yan L., Tei H., Moriya T., Shibata S., Loros J.J., Dunlap J.C. and Okamura H., Light-induced resetting of a mammalian circadian clock is associated with rapid induction of the mPerl transcript. Cell 91: 1043-1053, 1997.

9. Hardin P.E., Hall J.C. and Rosbash M. Feedback of the drosophila period gene product on circadian cycling of its messenger RNA levels. Nature 343: 536-540, 1990.

10. Gekakis N., Staknis D., Nguyen H.B., Davis F.C., Wilsbacher L.D., King D.P., Tahahashi J.S. and Weitz C.J., Role of the CLOCK protein in the mammalian circadian mechanism. Science 288: 1564-1569, 1998.

11. Jin X., Shearman L.P., Weaver D.R., Zylka M.J., de Vries G.J. and Reppert S.M., A molecular mechanism regulating rhythmic output from the suprachiasmatic nucleus. Cell 96: 57-68, 1999.

12. Kume K., Zylka M.J., Sriram S., Shearman L.P., Weaver D.H., Jin X., Maywood E., Hastings M.H. and Reppert S.M., mCRY1 and mCRY2 are essential components of the negative limb of the circadian clock feedback loop. Cell 98: 193-205, 1999.

13. Sangoram A.M., Saez L., Antoch M.P., Gekakis N., Staknis D., Whiteley A., Fruechte E.M., Vitaterna M.H., Shimomura K., King D.P., Young M.W., Weitz C.J. and Takahashi J.S., Mammalian circadian autoregulatory loop: a timeless ortholog and mPer1 interact and negatively regulate CLOCK-BMAL1-induced transcription. Neuron 21: 1101-1113, 1998.

14. Yamaguchi S., Mitsui S., Yan L., Yagita K., Miyake S. and Okamura H. Role of DBP in the circadian oscillatory mechanism. Mol. Cell Biol. 20: 4773-4781, 2000.

15 Shieh K.R., Distribution of the rhythm-related genes, rPeriod 1, rPeriod 2 , and $r$ Clock, in the rat brain. Neuroscience 118: 831-843, 2003.

16. Yan L. and Okamura H., Gradients in the circadian expression of Per1 and Per2 genes in the rat suprachiasmatic nucleus. Eur. J. Neurosci. 15: 1153-1162, 2002.

17. Yan L., Takekida S., Shigeyoshi Y. and Okamura H. Per 1 and Per2 gene expression in the rat suprachiasmatic nucleus: circadian profile and the compartment-specific response to light. Neuroscience 94: 141-150, 1999. 
18. Cheng M.Y., Bullock C.M., Li C., Lee A.G., Bermak J.C., Belluzzi J., Weaver D.R., Leslie F.M. and Zhou Q.Y., Prokineticin 2 transmits the behavioural circadian rhythm of the suprachiasmatic nucleus. Nature 417: 405-410, 2002.

19. Kramer A., Yang F.C., Snodgrass P., Li X., Scammell T.E., Davis F.C. and Weitz C.J., Regulation of daily locomotor activity and sleep by hypothalamic EGF receptor signaling. Science 294: 2511-2515, 2001.

20. Silver R., LeSauter J., Tresco P.A. and Lehman M.N., A diffusible coupling signal from the transplanted suprachiasmatic nucleus controlling circadian locomotor rhythms. Nature 382: 810-813, 1996.

21. Abe H., Honma S., Namihira M., Masubuchi S. and Honma K.I., Behavioural rhythm splitting in the CS mouse is related to clock gene expression outside the suprachiasmatic nucleus. Eur. J. Neurosci. 14: 1121-1128, 2001.

22. Bunger M.K., Wilsbacher L.D., Moran S.M., Clendenin C., Radcliffe L.A., Hogenesch J.B., Simon M.C., Takahashi J.S. and Bradfield C.A., Mop3 is an essential component of the master circadian pacemaker in mammals. Cell 103: 1009-1017, 2000.

23. Hamada T., LeSauter J., Venuti J.M. and Silver R., Expression of Period genes: rhythmic and nonrhythmic compartments of the suprachiasmatic nucleus pacemaker. J. Neurosci. 21: 7742-7750, 2001.

24. Kuhlman S.J., Quintero J.E. and McMahon D.G., GFP fluorescence reports Period 1 circadian gene regulation in the mammalian biological clock. Neuroreport 11: 1479$1482,2000$.

25. Masubuchi S., Honma S., Abe H., Ishizaki K., Namihira M., Ikeda M. and Honma K., Clock genes outside the suprachiasmatic nucleus involved in manifestation of locomotor activity rhythm in rats. Eur. J. Neurosci. 12: 4206-4214, 2000.

26 Maywood E.S., Mrosovsky N., Field M.D. and Hastings M.H., Rapid down-regulation of mammalian period genes during behavioral resetting of the circadian clock. Proc. Natl. Acad. Sci. USA 96: 15211-15216, 1999.

27 Preitner N., Damiola F., Luis L.M., Zakany J., Duboule D., Albrecht U. and Schibler U., The orphan nuclear receptor REV-ERB alpha controls circadian transcription within the positive limb of the mammalian circadian oscillator. Cell 110: 251-260, 2002.

28. Shearman L.P., Zylka M.J., Weaver D.R., Kolakowski L.F. and Reppert S.M., Two period homologs: circadian expression and photic regulation in the suprachiasmatic nuclei. Neuron 19: 1261-1269, 1997.

29. Takumi T., Matsubara C., Shigeyoshi Y., Taguchi K., Yagita K., Maebayashi Y., Sakakida Y., Okumura K., Takashima N. and Okamura H., A new mammalian period gene predominantly expressed in the suprachiasmatic nucleus. Gene. Cells 3: 167-176, 1998.

30. von Gall C., Garabette M.L., Kell C.A., Frenzel S., Dehghani F., Schumm-Draeger P.M., Weaver D.R., Korf H.W., Hastings M.H. and Stehle J.H., Rhythmic gene expression in pituitary depends on heterologous sensitization by the neurohormone melatonin. Nat. Neurosci. 5: 234238, 2002.

31. Wilsbacher L.D., Yamazaki S., Herzog E.D., Song E.J., Radcliffe L.A., Abe M., Block G., Spitznagel E., Menaker M. and Takahashi J.S., Photic and circadian expression of luciferase in mPeriodl-luc transgenic mice in vivo. Proc. Natl. Acad. Sci. USA 99: 489-494, 2002.

32. Yamazaki S., Numano R., Abe M., Hida A., Takahashi R., Ueda M., Block G.D., Sakaki Y., Menaker M. and Tei
$\mathrm{H}$. Resetting central and peripheral circadian oscillators in transgenic rats. Science 288: 682-685, 2000.

33. Zylka M.J., Shearman L.P., Weaver D.R. and Reppert S.M., Three period homologs in mammals: differential light responses in the suprachiasmatic circadian clock and oscillating transcripts outside of brain. Neuron 20: 11031110, 1998.

34. Lincoln G.A., Andersson H. and Hazlerigg D., Clock genes and the long-term regulation of prolactin secretion: evidence for a photoperiod/circannual timer in the pars tuberalis. J. Neuroendocrinol. 15: 390-397, 2003.

35. Lincoln G.A., Messager S., Andersson H. and Hazlerigg D., Temporal expression of seven clock genes in the suprachiasmatic nucleus and the pars tuberalis of the sheep: evidence for an internal coincidence timer. Proc. Natl. Acad. Sci. USA 99: 13890-13895, 2002.

36. Messager S., Garabette M.L., Hastings M.H. and Hazlerigg D.G., Tissue-specific abolition of Perl expression in the pars tuberalis by pinealectomy in the Syrian hamster. Neuroreport 12: 579-582, 2001.

37. Messager S., Hazlerigg D.G., Mercer J.G. and Morgan P.J., Photoperiod differentially regulates the expression of Per1 and ICER in the pars tuberalis and the suprachiasmatic nucleus of the Siberian hamster. Eur. J. Neurosci. 12: 2865-2870, 2000.

38. Sumova A., Jac M., Sladek M., Sauman I. and Illnerova $\mathrm{H}$. Clock gene daily profiles and their phase relationship in the rat suprachiasmatic nucleus are affected by photoperiod. J. Biol. Rhythms 18: 134-144, 2003.

39 Messager S., Ross A.W., Barrett P. and Morgan P.J., Decoding photoperiodic time through Perl and ICER gene amplitude. Proc. Natl. Acad. Sci. USA 96: 9938-9943, 1999.

40. Dardente H., Menet J.S., Poirel V.J., Streicher D., Gauer F., Vivien-Roels B., Klosen P., Pevet P. and Masson-Pevet M., Melatonin induces Cryl expression in the pars tuberalis of the rat. Brain Res. Mol. Brain Res. 114: 101-106, 2003

41. Abe M., Herzog E.D., Yamazaki S., Straume M., Tei H., Sakaki Y., Menaker M. and Block G.D., Circadian rhythms in isolated brain regions. J. Neurosci. 22: 350 356, 2002.

42. Kriegsfeld L.J., Korets R. and Silver R., Expression of the circadian clock gene Period 1 in neuroendocrine cells: an investigation using mice with a Per $1::$ GFP transgene. Eur. J. Neurosci. 17: 212-220, 2003.

43. Shieh K.R. and Pan J.T., An endogenous cholinergic rhythm may be involved in the circadian changes of tuberoinfundibular dopaminergic neuron activity in ovariectomized rats treated with or without estrogen. Endocrinology 136: 2383-2388, 1995.

44. Shieh K.R. and Pan J.T., Sexual differences in the diurnal changes of tuberoinfundibular dopaminergic neuron activity in the rat: role of cholinergic control. Biol. Reprod. 54: 987-992, 1996.

45. Shieh K.R., Chu Y.S. and Pan J.T., Circadian change of dopaminergic neuron activity: effects of constant light and melatonin. Neuroreport 8: 2283-2287, 1997.

46 Morse D. and Sassone-Corsi P. Time after time: inputs to and outputs from the mammalian circadian oscillators. Trends Neurosci. 25: 632-637, 2002.

47. Lu X.Y., Shieh K.R., Kabbaj M., Barsh G.S., Akil H. and Watson S.J., Diurnal rhythm of agouti-related protein and its relation to corticosterone and food intake. Endocrinology 143: 3905-3915, 2002. 